\title{
Endoscopic transpterygoid approach to the skull base lesions
}

\author{
Sang-Chul Lim, M.D.
}

Chonnam National Universtiy Medical School, Korea

Endoscopic transpterygoid approaches have recently provided new corridors to access a variety of pathologies in the paramedian and lateral skull base. An endoscopic transpterygoid approach was first described to access the lateral recess of the sphenoid sinus and was subsequently modified and expanded to manage lesions of the petrous apex, middle cranial fossa, and infratemporal fossa. Common pathologies in the paramedian skull base lesions include schwannnoma, chondrosarcoma, CSF leak, meningoencephalocele, meningioma, and various malignancies including nasopharyngeal carcinoma and adenoid cystic carcinoma. Understanding the complex anatomical relationships of the skull base including the pterygopalatine fossa and infratemporal fossa from the endoscopic perspective is mandatory before attempting an endoscopic transpterygoid approach. Endoscopic removal of the skull base lesions with transpterygoid approach require a large mid-meatal nasoantral window, a medial maxillectomy or even an endoscopic Denker's approach to expose the entire posterior wall of the antrum. After the posterior wall of the maxillary sinus is removed, the pterygopalatine fossa is open. The soft tissues of the pterygopalatine fossa are mobilized laterally, exposing the vidian canal, vidian nerve and the foramen rotundum and V2 over the anterior and superior aspect of the pterygoid process. Proper identification and preservation of these landmarks are followed by the opening of the lateral recess of the sphenoid sinus and drilling of the base of the pterygoid process; thus, allowing a direct access to the medial aspect of the infratemporal fossa and middle cranial fossa. Here, the cases from Chonnam national university hospital are presented and treatment outcome is discussed. 


\section{A novel endoscopic approach for nasal tumors \\ —Trans-septal Access with Crossing Multiple Incisions (TACMI)—}

Kazuhiro Omura ${ }^{1}$, Nobuyoshi Otori ${ }^{2}$, Satoshi Aoki ${ }^{1}$, Keisuke Miyashita $^{1}$, Yu Hosokawa ${ }^{1}$, and Yasuhiro Tanaka ${ }^{1}$

${ }^{1}$ Department of Otorhinolaryngology, Dokkyo Medical University Koshigaya Hospital, Saitama, Japan

${ }^{2}$ Department of Otorhinolaryngology, The Jikei University School of Medicine, Tokyo, Japan

Introduction:

Recently the surgical management of nasal tumors has been greatly influenced by advanced endoscopic techniques. To achieve complete endoscopic resection of large obstructing nasal tumors, a sufficient corridor for adequate exposure is also required. In this report, we describe an endoscopic technique with novel elements that we term Trans-septal Access with Crossing Multiple Incisions (TACMI), which enables a direct trans septal approach to the pedicle through tumor transposition with complete septum reconstruction. In the Japanese language, TACMI means "Master Expert".

Methods:

We prospectively collected data from 30 patients who underwent TACMI approach for sinonasal tumors.

Results:

Using TACMI, complete en bloc neoplasm resection was achieved in all but 2 patients, who underwent piecemeal tumor removal. The follow-up duration ranged from 2 to 28 months. None of the patients experienced septal perforation or tumor recurrence.

\section{Conclusions:}

We show evidence that TACMI is a simple, efficient and effective technique for sinonasal tumor transposition, and visualization of, and access to, the pedicle of challenging unilateral sinonasal lesions. These features contribute to limited blood loss, better pedicle control with complete nasal valve and septum preservation without functional concerns. 


\title{
Role of Gut Microbiome in the Pathogenesis of Allergic Airway Disease
}

\author{
Chang-Hoon Kim, MD
}

Department of Otorhinolaryngology

The Airway Mucus Institute

Korea Mouse Sensory Phenotyping Center (KMSPC)

Taste Research Center (TRC/MRC)

Yonsei University College of Medicine, Korea

Gut microbiota and the host have a symbiotic relationship, whereas altered gut microbiota has been associated with immune-mediated diseases, including allergic airway diseases. The first colonizers are derived from the mother before and during delivery and there is emerging evidence of an early-life critical window, when the effects of gut microbial dysbiosis are most influential in immune development at the mucosal surfaces. Here we sought to further clarify the pathophysiology of allergic airway diseases by determining whether perinatal antibiotic treatment has altered of gut microbiota and regulates susceptibility to a TH2 model of allergic asthma. Allergic asthma was induced BALB/c wild-type mice treated perinatally with ampicillin, vancomycin, and metronidazole in drinking water. Disease severity was assessed by measuring lung inflammation, pathology, cytokine response, and serum antibodies. Microbial community analyses were performed on stool samples via Next Generation Sequencing. We found that perinatal triple antibiotics treatment profoundly altered the gut microbial composition and were not observed to develop phenotype of allergic asthma. Perinatal antigen exposure of mice elicited a splenomegaly that was induced by neutrophils in spleen. Interestingly, we showed a decreased in dendritic cells of lung which reside in asthmatic neonatal group. Our findings will inform the development of novel approaches to investigate the relationship lung-gut axis based on modulating the composition of the gut microbiota. 


\section{Epithelial cell-basophil interactions stimulate the production of IL-5 in innate allergic inflammation.}

Koji Matsumoto, Hideaki Kouzaki, Ichiro Tojima, Takeshi Shimizu

Department of Otorhinolaryngology, Shiga University of Medical Science, Japan

Introduction: Epithelial cell-derived interleukin (IL)-33 plays an important role in the initiation and activation of innate allergic inflammation by stimulating the production of Th2 cytokines. IL-33 acts as a cytokine through the transmembrane receptor ST2L, which is expressed in most of the inflammatory cells, and its activity is neutralized by the soluble spliced variant of ST2 (sST2). Basophil expresses ST2L and produces IL-5 in response to IL-33, however, the role of epithelial cell-basophil interactions in allergic inflammation is still unclear.

Method: Cultured human bronchial epithelial (hBE33) cells that were introduced human IL-33 gene into normal human bronchial epithelial cells and human basophil cell line KU812 cells were used to study the epithelial cell-basophil interactions in the house dust mite (HDM)-induced production of IL-5. Production of IL5, IL-33 and sST2 was evaluated by ELISA.

Results: HDM stimulated the rapid release of IL-33 from cultured hBE33 cells at 1 hour after the incubation. Basophil cell line KU812 cells produced IL-5 in response to IL-33, and the conditioned medium of HDMstimulated hBE33 cells stimulated IL-5 production from KU812 cells. However, HDM did not stimulate IL-5 production from co-cultured KU812 cells with hBE33 cells, and HDM stimulated the production of sST2 timedependently in co-cultured cells. IL-33 antibody, ST2 antibody and recombinant sST2 suppressed IL-5 production from KU812 cells incubated with the conditioned medium of HDM-stimulated hBE33 cells.

Conclusion: These results indicate that epithelial cell-derived IL-33 stimulates the production of IL-5 from basophils, and sST2 is an important regulator of IL-33 in innate allergic inflammation. 